Article

\title{
Ti-doped ZnO Thin Films Prepared at Different Ambient Conditions: Electronic Structures and Magnetic Properties
}

Zhihua Yong ${ }^{1,4}$, Tao Liu ${ }^{1,2, *}$, Tomoya Uruga ${ }^{3}$, Hajime Tanida ${ }^{3}$, Dongchen Qi ${ }^{1,4}$, Andrivo Rusydi ${ }^{1,4,5}$ and Andrew T. S. Wee ${ }^{1}$

1 Physics Department, Faculty of Science, National University of Singapore, 117542, Singapore

2 Institute for Synchrotron Radiation, Karlsruhe Institute of Technology, 76344, Germany

3 SPring-8/JASRI, Hyogo, 679-5198, Japan

4 NanoCore, National University of Singapore, 117576, Singapore

5 Singapore Synchrotron Light Source, National University of Singapore, 117603, Singapore

* Author to whom correspondence should be addressed; E-Mail: tao.liu@kit.edu.

Received: 20 April 2010; in revised form: 27 May 2010 / Accepted: 3 June 2010 /

Published: 9 June 2010

\begin{abstract}
We present a comprehensive study on Ti-doped ZnO thin films using X-ray Absorption Fine Structure (XAFS) spectroscopy. Ti K edge XAFS spectra were measured to study the electronic and chemical properties of $\mathrm{Ti}$ ions in the thin films grown under different ambient atmospheres. A strong dependence of Ti speciation, composition, and local structures upon the ambient conditions was observed. The XAFS results suggest a major tetrahedral coordination and a 4+ valence state. The sample grown in a mixture of $80 \%$ Ar and $20 \% \mathrm{O}_{2}$ shows a portion of precipitates with higher coordination. A large distortion was observed by the $\mathrm{Ti}$ substitution in the $\mathrm{ZnO}$ lattice. Interestingly, the film prepared in $80 \% \mathrm{Ar}, 20 \% \mathrm{O}_{2}$ shows the largest saturation magnetic moment of $0.827 \pm 0.013 \mu_{B} / \mathrm{Ti}$.
\end{abstract}

Keywords: DMS; XAFS; ZnO; vacancy; Ti

\section{Introduction}

Spintronics, the manipulation of spin in electrons in semiconductors presents a new paradigm for versatile functionalities in electronic materials. Ferromagnetic semiconductors with Curie temperatures $\left(T_{C}\right)$ above room temperature are ideal for realizing efficient spintronics devices. Dilute magnetic 
semiconductors (DMS) are promising materials since they have both charge and spin degrees of freedom in a single matrix, leading to interplay of magnetic, optical, and electronic functionalities. However, the origin of room temperature ferromagnetism in DMS is still controversial. Many mechanisms have been proposed so far. For example, the carrier-induced ferromagnetism which includes the RKKY (Ruderman-Kittel-Kasuya-Yosida) model [1,2], the double exchange mechanism [3], and more recently, Coey et al. [4,5] and Chambers et al. [6] reported that the phantom ferromagnetism can result from structural defects. Coey et al. [7] and Venkatesan et al. [8] proposed the bound magnetic polaron model [9], which states that ferromagnetic exchange is mediated by shallow donor electrons, which form bound magnetic polarons. Interestingly, as proposed by Elfimov et al. [10] and Jorge et al. [11], cationic vacancies can also lead to ferromagnetism. The experimentally observed ferromagnetism is often plagued by possible magnetic precipitates or clusters in the host semiconductors, casting doubts upon the reproducibility and reliability of current DMSs. Hence, careful studies on the electronic and atomic structures of DMS are essential to identifying the origin of the ferromagnetism in DMS for future fabrication of high $\mathrm{T}_{\mathrm{C}}$ ferromagnetic DMSs.

Semiconducting zinc oxide $(\mathrm{ZnO})$ offers significant potential in providing charge, photonic, and spins based functionalities [12]. ZnO is also well known for its direct band-gap and large excitation energy, and exhibits unique piezoelectric and electro-optic properties with potential applications in UV photonics and transparent electronics. Since $\mathrm{ZnO}$ has been identified as a good host material for realizing wide band-gap DMS with high $T_{C}$ ferromagnetism by doping with transition metal ions, $\mathrm{ZnO}$ has been selected as the host material in our investigation. In particular, Ti-doped $\mathrm{ZnO}$ seems to be a promising DMS material [8,13,14], since neither Ti nor its oxides are ferromagnetic. However, previous reports on the magnetic properties of $\mathrm{ZnO}$ :Ti are inconsistent; the theoretical value of the magnetic moment of Ti doped in $\mathrm{ZnO}$ is predicted to be 0 by Sato et al. [15] However, Antony et al. [13] and Venkatesan et al. [8] reported a saturation moment of about $0.15 \mu_{B} / \mathrm{Ti}$ atom at room temperature for 5\% Ti-doped $\mathrm{ZnO}$ sample, and Osuch et al. [14] performed density functional theory calculations predicting a magnetic moment of $0.63 \mu_{B}$ per supercell in $\mathrm{Zn}_{0.9375} \mathrm{Ti}_{0.0625} \mathrm{O}$.

$\mathrm{X}$-ray absorption fine structure (XAFS) measurements reveal the absorption characteristics of $\mathrm{X}$-rays absorbed by an atom at energies near and above the core-level binding energies of that atom. XAFS spectra give information on the immediate environment around each absorbing species, and are especially sensitive to the formal oxidation state, coordination chemistry, interatomic distances, coordination number, and the species of atoms immediately surrounding the selected element. As XAFS provides a practical and relatively simple way to determine the chemical state and local atomic structure for selected atomic species, we make full use of this technique to investigate the nature of the Ti ions doped in the $\mathrm{ZnO}$ matrix.

In this work, Ti-doped $\mathrm{ZnO}$ thin films were prepared at several ambient conditions and the structure, electronic state, composition, and chemical environment were investigated. The composition of the prepared films depends on the ambient atmospheres because of the different deposition rates. From the results, a possible link between the structural and electronic properties is explored and the mechanism leading to ferromagnetism in Ti-doped $\mathrm{ZnO}$ films is discussed. 


\section{Experimental Section}

Ti-doped $\mathrm{ZnO}$ thin films were fabricated by a reactive radio-frequency (RF) magnetron sputtering from a commercial $\mathrm{ZnO}$ target of $99.995 \%$ purity with a small plate of pure Ti metal plate attached. A $\mathrm{ZnO}$ target is selected instead of a $\mathrm{Zn}$ metallic target because the control of film stoichiometry is easier with oxide targets, thus alleviating the need for high temperature and post-deposition annealing [16].

A $\mathrm{ZnO}$ buffer layer was first grown between the Si(100) substrate and $\mathrm{ZnO}$ film to reduce the lattice mismatch between $\mathrm{ZnO}$ and $\mathrm{Si}(100)$. Next, the $\mathrm{ZnO}$ layer was deposited at a higher temperature on the buffer layer to obtain a high-quality thin film. Reports [17-19] have shown that there is marked improvement in both the optical properties and crystalline quality achieved through a two-step growth using the RF magnetron sputtering system.

In the experiments, the chamber was first pumped down from $10^{-6}$ to $10^{-7}$ Torr and the voltage for sputtering was set at $28 \mathrm{~V}$. The target was first pre-sputtered for 6 to 8 min for surface cleanness at $200{ }^{\circ} \mathrm{C}$. Buffer layers were then created on the $\mathrm{Si}(100)$ substrates at $200{ }^{\circ} \mathrm{C}$ for $2 \mathrm{~min}$ at a deposition power of $150 \mathrm{~W}$. The temperature of the heater was then raised to $400^{\circ} \mathrm{C}$. The target was pre-sputtered again for $3 \mathrm{~min}$ at $400{ }^{\circ} \mathrm{C}$ before a further $60 \mathrm{~min}$ of sputtering at $400{ }^{\circ} \mathrm{C}$ and at $150 \mathrm{~W}$; thin films of Ti-doped $\mathrm{ZnO}$ at a certain thickness were formed. The films were deposited in high vacuum at different ambient atmospheres at a pressure of 1.0 2.0 mTorr: (1) a mixture of 85\% Ar and 15\% $\mathrm{N}_{2}$, (2) a mixture of $80 \% \mathrm{Ar}$ and $20 \% \mathrm{O}_{2}$, and (3) $100 \% \mathrm{Ar}$. The gas flow was set at $18.7 \sim 21.0 \mathrm{sccm}$ (atm $\mathrm{cm}^{3} / \mathrm{min}$ ). The target to substrate distance was fixed throughout the experiment. Two sets of samples were prepared at the $85 \%$ Ar and $15 \% \mathrm{~N}_{2}$ mixture ambient. The concentration of Ti doped in the doped samples was varied by changing the Ti plate size.

The thickness of Ti-doped $\mathrm{ZnO}$ films was measured using an Alpha-step 500 profilometer, revealing a thickness of approximately $500 \mathrm{~nm}$. Scanning electron microscopy (SEM) images show a polycrystalline nature of the film surfaces, which have relatively small and slightly elongated spherical grain structures. X-ray fluorescence (XRF) analysis was also performed using white beam radiation at the Phase Contrast Imaging (PCI) beamline [20] at the Singapore Synchrotron Light Source (SSLS) to determine the composition of samples, as listed in Table $1 . \mathrm{Zn}_{0.996} \mathrm{Ti}_{0.004} \mathrm{O}$ and $\mathrm{Zn}_{0.972} \mathrm{Ti}_{0.028} \mathrm{O}$ were fabricated under $85 \%$ Ar and $15 \% \mathrm{~N}_{2}$, and $\mathrm{Zn}_{0.933} \mathrm{Ti}_{0.067} \mathrm{O}$ and $\mathrm{Zn}_{0.994} \mathrm{Ti}_{0.006} \mathrm{O}$ were fabricated under pure $\mathrm{Ar}$, and $80 \% \mathrm{Ar}$ and $20 \% \mathrm{O}_{2}$, respectively.

Table 1. Composition, saturation magnetic moments $\mathrm{M}_{\mathrm{s}}$ of Ti-doped $\mathrm{ZnO}$ films deposited on $\mathrm{Si}(100)$ substrate and the ambient atmosphere under which the films were fabricated.

\begin{tabular}{ccc}
\hline Sample & Atmosphere & $\mathbf{M}_{\mathbf{s}} / \boldsymbol{\mu}_{\boldsymbol{B}}$ per Ti atom \\
\hline $\mathrm{Zn}_{0.996} \mathrm{Ti}_{0.004} \mathrm{O}$ & $\mathrm{Ar}+\mathrm{N}_{2}$ & $0.090 \pm 0.004$ \\
$\mathrm{Zn}_{0.994} \mathrm{Ti}_{0.006} \mathrm{O}$ & $\mathrm{O}_{2}+\mathrm{N}_{2}$ & $0.827 \pm 0.013$ \\
$\mathrm{Zn}_{0.933} \mathrm{Ti}_{0.067} \mathrm{O}$ & $\mathrm{Ar}$ & $0.036 \pm 0.001$ \\
$\mathrm{Zn}_{0.972} \mathrm{Ti}_{0.028} \mathrm{O}$ & $\mathrm{Ar}+\mathrm{N}_{2}$ & $0.039 \pm 0.002$ \\
\hline
\end{tabular}

The XAFS spectra of the Ti-doped ZnO films were measured at the X-ray Development and Demonstration (XDD) beamline [21] at SSLS. The XDD beamline provides a photon energy range of 2.4-10 keV from a superconducting bending magnet. The XAFS spectra of reference samples were taken in transmission mode at room temperature. The film samples were measured in fluorescence 
mode using a Lytle-type detector with argon ambient flowing. Because of the low concentration and lower binding energy of $\mathrm{Ti}$ with respective to $\mathrm{Zn}$, Ti K-edge fluorescence XAFS experiments were also repeated at the BL01B1 beamline [22,23] of SPring-8 using a 19-element solid state detector [24]. BL01B1 is a bending magnet beamline equipped with two mirrors and a fixed-exit double-crystal monochromator using $\mathrm{Si}(111)$ and $\mathrm{Si}(311)$ crystals. The energy was calibrated to the K-edge absorption of the Ti metal-foil. XAFS spectra were collected in the photon energy range from roughly $100 \mathrm{eV}$ prior to the absorption edge to $800 \mathrm{eV}$ above. The data presented here were collected at Spring-8.

Data analysis of XAFS follows a standard procedure. Because of the data statistics, only the two high $\mathrm{Ti}$ concentration samples were subject to the Fourier transform (FT). In the FT, the XAFS functions in a range of $2.9-11.0 \AA^{-1}$ in $k$ space were extracted, $\mathrm{k}^{3}$ weighted, and a Bessel window function was employed. A fit to the first shell was performed in the real space and the coordination number $(\mathrm{CN})$, interatomic distance $(\mathrm{R})$, and Debye-Waller $\left(\sigma^{2}\right)$ factor were extracted. $E_{0}$ values were set as free variables during the fit. The inelastic factor, $s_{0}{ }^{2}$, was fixed at 0.90 .

A model 707 vibrating sample magnetometer produced by the Lake-Shore Company was used in the magnetic measurements of the film samples. The magnetic field was applied parallel to the plane of the film. Before the measurements, the sample holder with pure Si substrate was measured to calibrate the magnetic background. Magnetic contaminations by sample handling were avoided. A maximum of 5000 Gaus magnetic field was applied. All magnetization curves were measured at room temperature.

\section{Results and Discussion}

The penetration depth of the hard X-rays used in XRF is in the $\mu \mathrm{m}$ range; hence, XRF probes the bulk of the films. Figure 1 shows the normalized XRF spectra of the Ti-doped ZnO samples. The fluorescent peaks of $\mathrm{Ti}$ and $\mathrm{Zn}$ are clearly observable. The peak of argon at about $3 \mathrm{keV}$ is from the rare gas since the samples were measured in air. The $\mathrm{Cr}$ and $\mathrm{Fe}$ signals are from the background contamination which contains stainless steel. By comparing the integrated areas under the $\mathrm{Zn} \mathrm{K}_{\alpha}$ and $\mathrm{Ti} \mathrm{K}_{\alpha}$ peaks and comparing with a calibrated sample of known concentration, the composition $\mathrm{x}$ of $\mathrm{Ti}$ was derived, as listed in Table 1.

$\mathrm{X}$-ray diffraction (XRD) patterns were measured for the Ti-doped $\mathrm{ZnO}$ films (Figure 2), which show only (002) and (004) index peaks of $\mathrm{ZnO}$, indicating that $\mathrm{ZnO}$ grains are preferentially c-axis oriented on $\mathrm{Si}(100)$. A strong crystallographic anisotropy is observed in the measured XRD patterns. No metal or metal oxide related peaks can be detected within the sensitivity of XRD. The peak position of $\mathrm{ZnO}$ :Ti is shifted by $0.1^{\circ}-0.2^{\circ}$ towards higher angles as compared to the undoped $\mathrm{ZnO}$, which implies a decreased lattice parameter c for the Ti-doped $\mathrm{ZnO}$ films. The decrease in c is in agreement with the previous theoretical calculations [14]. This might be due to the atomic radius difference for Ti and Fe ions and complex defect formation [25,26]. 
Figure 1. Normalized X-ray fluorescence spectra of the $\mathrm{Zn}_{1-x} \mathrm{Ti}_{x} \mathrm{O}$ films deposited on $\mathrm{Si}(100)$. The Ti compositions $\mathrm{x}$ relative to $\mathrm{Zn}$ were determined. The Fe and $\mathrm{Cr}$ peaks in the spectra are contaminants from the background that contain stainless steel and can be removed by careful shielding of radiation.

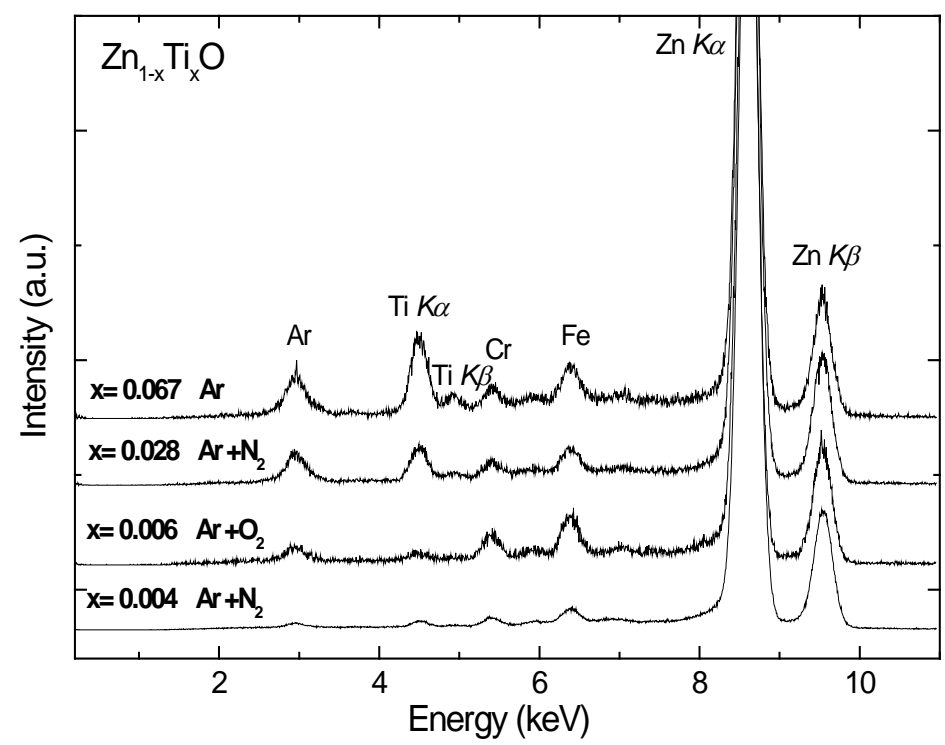

Figure 2. X-ray diffraction patterns of the $\mathrm{Zn}_{1-x} \mathrm{Ti}_{x} \mathrm{O}$ films deposited on $\mathrm{Si}(100)$.

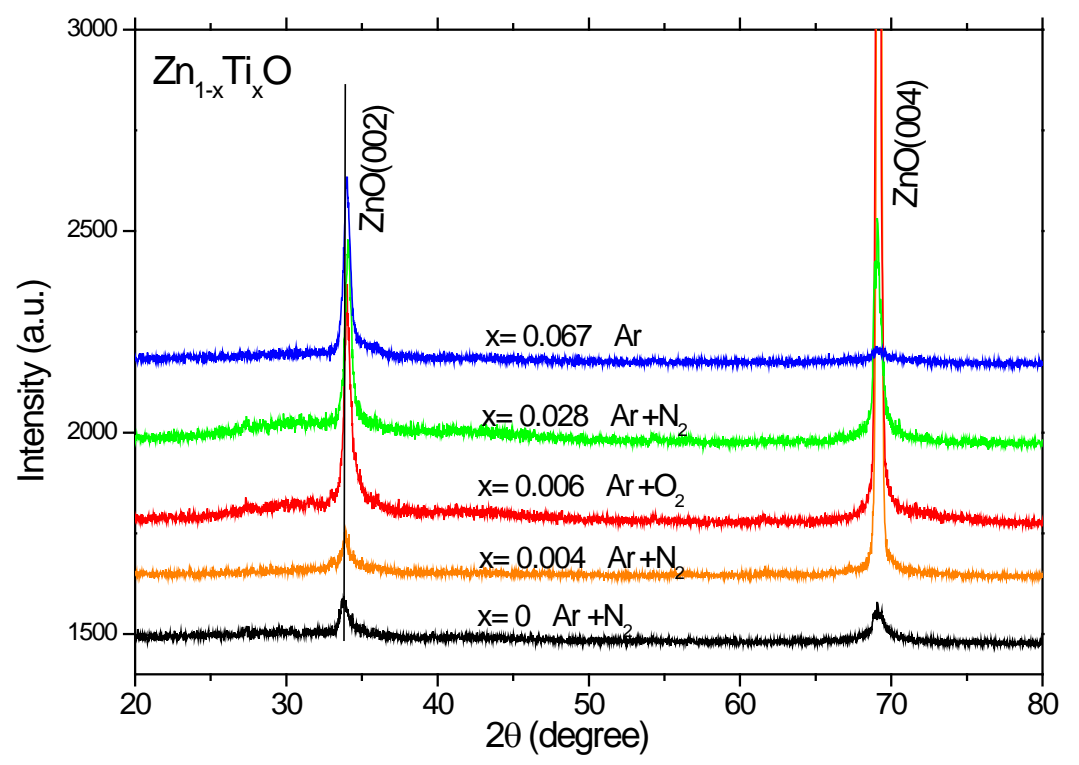

Figure 3 compares the normalized Ti K-edge X-ray absorption near-edge spectroscopy (XANES) spectra of the Ti-doped $\mathrm{ZnO}$ films with those of $\mathrm{Ti}$ foil, $\mathrm{Ti}_{2} \mathrm{O}_{3}$, and $\mathrm{TiO}_{2}$. Several main features are identified and marked: a very sharp pre-edge peak A, a shoulder peak B, a main peak $\mathrm{C}$, and a broad peak $\mathrm{D}$ at the post-edge region. Generally, the peak A for transitional $3 d$ metal oxides is interpreted as a quadrupolar electronic transition from $1 s$ to the unoccupied $3 d$ final states hybridized with the $4 p$ character of the absorber; its intensity is enhanced by the local atomic configuration that lacks centrosymmetry $[27,28]$. 
Figure 3. Normalized Ti K-edge XANES of Ti-doped ZnO films and reference samples, Ti foil, $\mathrm{Ti}_{2} \mathrm{O}_{3}$ and $\mathrm{TiO}_{2}$.

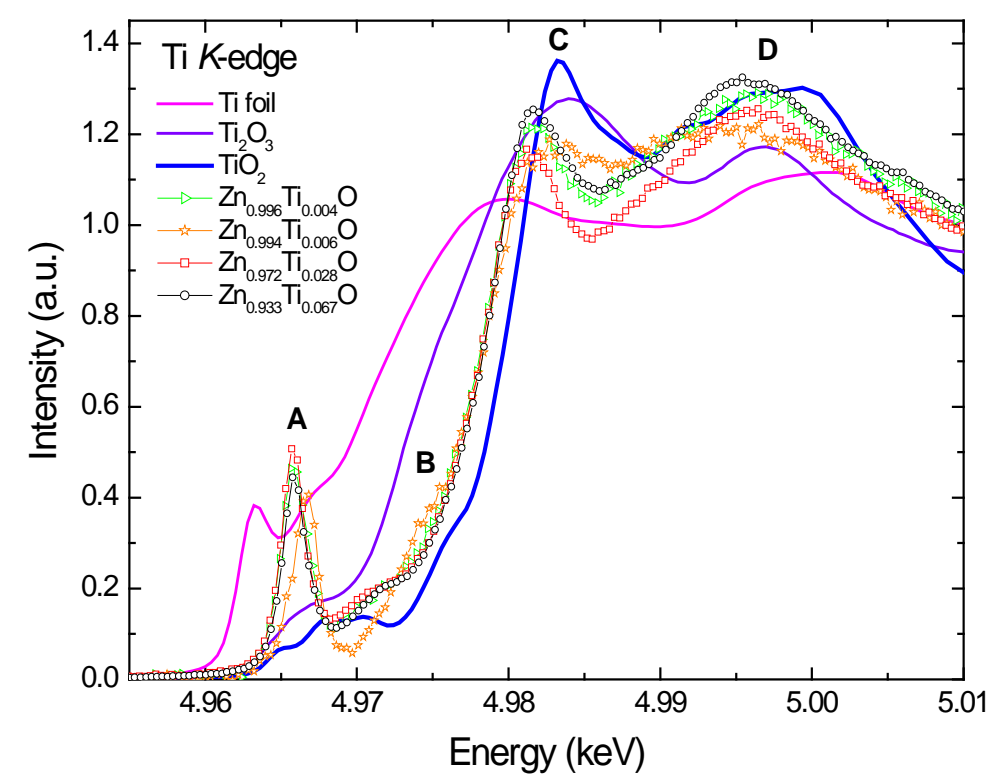

The XANES spectra of Ti doped ZnO samples display significantly different structures from the reference $\mathrm{Ti}$ foil and oxides. The very prominent pre-edge peak indicates a tetrahedral Ti-O coordination, implying a substitutional incorporation of $\mathrm{Ti}$ ions into the tetrahedral zinc sites in the wurzite $\mathrm{ZnO}$ lattice. The spectra of $\mathrm{Ti}_{2} \mathrm{O}_{3}$ and $\mathrm{TiO}_{2}$ show only a weak pre-edge peak A due to the octahedral local structures around $\mathrm{Ti}$ ions [29-31]. The pre-edge features are insensitive to Ti-O bond length, but are sensitive to valence, occurring about $2.0 \mathrm{eV}$ lower in $\mathrm{Ti}^{3+}$ samples compared to $\mathrm{Ti}^{4+}$ [32]. However, it is difficult to deduce unambiguously the Ti valence because the pre-edge features vary in both position ( $\sim 2 \mathrm{eV}$ ) and normalized height as a function of Ti coordination (4, 5 or 6 oxygen nearest neighbors) [33]. Ideally the Ti doped samples should be compared to the references with identical coordination and known valency. Few minerals of $\mathrm{Ti}$ in nature appear to have major $\mathrm{Ti}^{3+}[32]$.

There is an evident shift of the peak A to higher energy for $\mathrm{Zn}_{0.994} \mathrm{Ti}_{0.006} \mathrm{O}$ relative to the rest of the samples. Since the feature A is still predominant, it implies that the majority of $\mathrm{Ti}$ in $\mathrm{Zn}_{0.994} \mathrm{Ti}_{0.006} \mathrm{O}$ is still tetrahedrally coordinated. This chemical shift is either due to a change in the valence state of Ti to a higher oxidation state or due to the precipitation of a portion of octahedral Ti species. The slightly reduced peak height for this sample implies the latter case is more likely. Some other differences are also observed: the shoulder $\mathrm{B}$ is more pronounced, and the peak $\mathrm{C}$ broadened, with its intensity much lower. Although the Ti doping concentration is extremely low in this sample, a portion of titanium oxides or zinc titanate might have formed during the sputtering process. The Ti atoms sputtered from the target might have reacted actively with oxygen ions and formed oxide precipitates before the deposition. This would hinder them from incorporating into the ZnO lattice. Despite the similar deposition rate for the $\mathrm{x}=0.004$ and $\mathrm{x}=0.006$ samples, they are not prepared in the same ambient atmospheres. This leads to the different the species for Ti, indicating that the prepared Ti speciation in $\mathrm{ZnO}$ is sensitive to the ambient atmosphere. These trace precipitates containing octahedral Ti may be too dispersive and disordered, and are not detectable by XRD. 
Figure 4a and 4b show Ti K-edge EXAFS functions and Fourier transform of the $\mathrm{Zn}_{0.972} \mathrm{Ti}_{0.028} \mathrm{O}$ and $\mathrm{Zn}_{0.933} \mathrm{Ti}_{0.067} \mathrm{O}$ films and reference samples. The Ti K-edge EXAFS functions for the low concentration samples $\left(\mathrm{Zn}_{0.996} \mathrm{Ti}_{0.004} \mathrm{O}\right.$ and $\left.\mathrm{Zn}_{0.994} \mathrm{Ti}_{0.006}\right)$ are not usuable because of the poor statistics. The EXAFS functions for $\mathrm{Zn}_{0.972} \mathrm{Ti}_{0.028} \mathrm{O}$ and $\mathrm{Zn}_{0.933} \mathrm{Ti}_{0.067} \mathrm{O}$ are very similar and different from $\mathrm{Ti}$ oxides. In Figure 4b, the first peak at 1.4 1.5 $\AA$ (phase shift was not corrected) is due to the Ti-O (or Zn-O for $\mathrm{ZnO}$ ) coordinations in the first shell. The second peak at about 3.15 3.2 $\AA$ corresponds to the second shell, which contains $12 \mathrm{Zn}$ and $1 \mathrm{O}$ atoms for $\mathrm{ZnO}$ [34]. The second coordination peaks are weak for the Ti-doped samples, but not absent if one looks into the structures at an even higher R, which represents the level of noise. Even at such low Ti concentrations, an extreme disorder is possible because of the large difference in atomic radius of $\mathrm{Zn}^{2+}(0.600 \AA)$ and $\mathrm{Ti}^{4+}(0.42 \AA)$ in a tetrahedral Ti-O coordination [35]. A shift towards higher $R$ for the second Ti-Zn shell is also observed for the Ti doped samples relative to $\mathrm{ZnO}$.

Figure 4. (a; left): Ti K-edge EXAFS functions; (b; right): Fourier Transform magnitudes (vertically shifted) of the Ti-doped $\mathrm{ZnO}$ films and reference Ti oxide samples. Phase shift was not corrected.
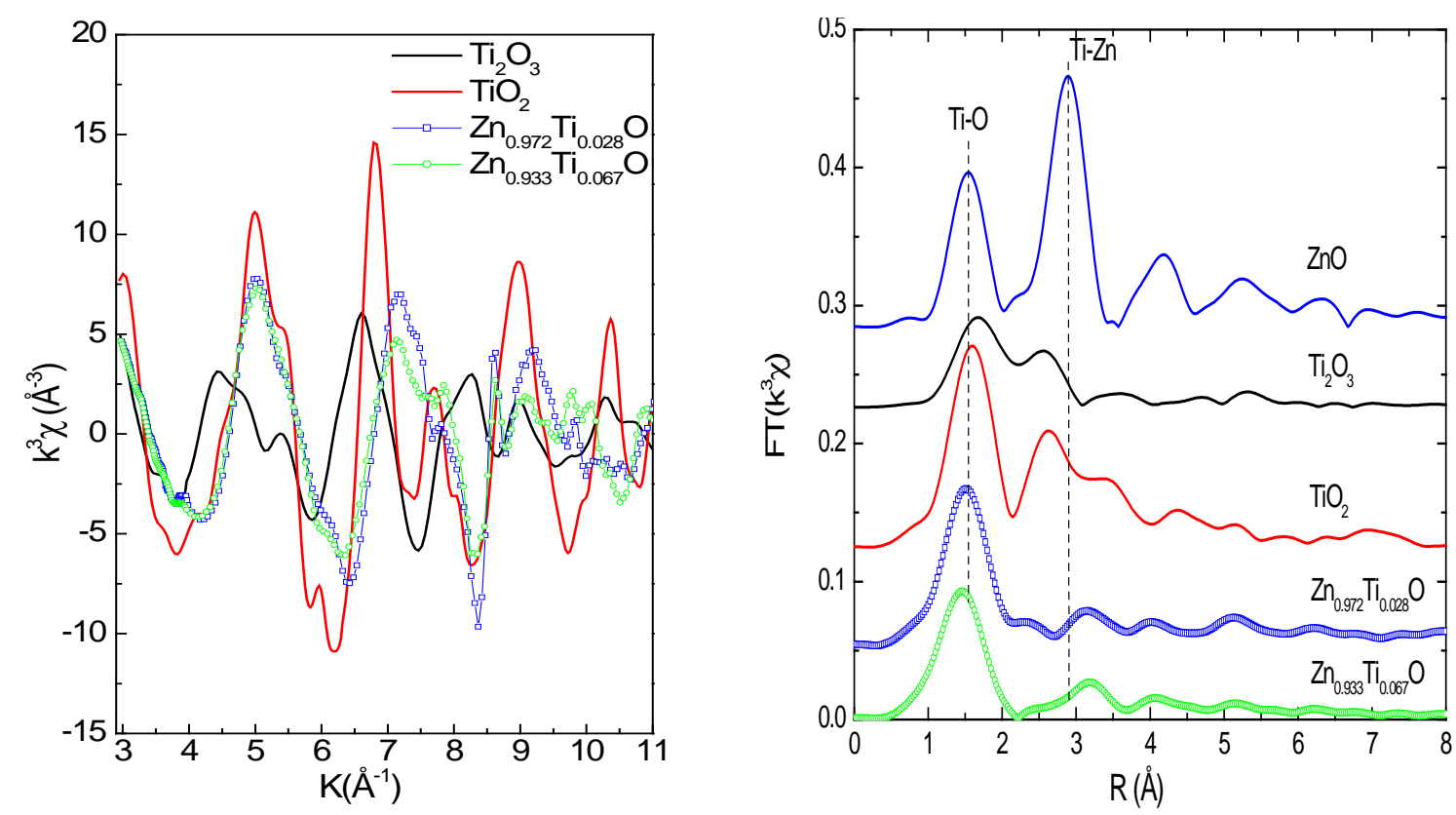

Data fit of the Ti-doped samples was performed in real space for the first shell (Figure 5); the results are tabulated in Table 2. The radial structural parameters for the reference samples are extracted from crystallographic data. The fit yields a Ti-O distance of 1.87-1.89 $\AA$ for the Ti doped $\mathrm{ZnO}$ samples. It is much less than those of $\mathrm{Zn}-\mathrm{O}(1.97 \AA)$ in $\mathrm{ZnO}$ and $\mathrm{Ti}-\mathrm{O}$ in $\mathrm{Ti}_{2} \mathrm{O}_{3}$ and $\mathrm{TiO}_{2}$, as listed in Table 2. This much shorter Ti-O bond favors the $4+$ valence and a tetrahedral coordination, since the difference in bond length for $\mathrm{Ti}^{3+}$ and $\mathrm{Ti}^{4+}$ is $0.05-0.1 \AA$ [32] and the atomic radius in tetrahedral sites is shorter than in octahedral sites [35]. The short Ti-O distance implies strong local interaction in Ti-O in the ZnO matrix. The empty $d$-orbitals of Ti might be involved in the chemical bonding. Similarly, a strong Co-O local interaction in Co-doped $\mathrm{ZnO}$ nanoparticles has been observed [36]. It is in agreement with our XRD results and the theoretical calculations performed by Xiong et al. [12]. The coordination number (CN) for $\mathrm{Zn}_{0.972} \mathrm{Ti}_{0.028} \mathrm{O}$ is close to 4 and it gets higher (5.4) for the $\mathrm{x}=0.067$ 
doped sample, implying a portion of precipitates with higher $\mathrm{CN}$ at the first shell may exist in the 6.7\% doped concentration. Two other possible zinc titanates that may form precipitates are $\mathrm{Zn}_{2} \mathrm{Ti}_{3} \mathrm{O}_{8}$ and $\mathrm{ZnTiO}_{3}$. The radial structures for the first shell are extracted from the crystallographic data and are also listed in Table 2. $\mathrm{Zn}_{2} \mathrm{Ti}_{3} \mathrm{O}_{8}$ is a metastable compound and possesses a defect spinel structure [37]. $\mathrm{Ti}^{4+}$ ions in $\mathrm{Zn}_{2} \mathrm{Ti}_{3} \mathrm{O}_{8}$ occupy octahedral sites, whereas part of the $\mathrm{Zn}^{2+}$ ions occupy octahedral sites and the rest are in tetrahedral sites. $\mathrm{ZnTiO}_{3}$ has a perovskite structure and is stabilized by the six-fold coordination of the $\mathrm{Ti}^{4+}$ ions and 12-fold coordination of the $\mathrm{Zn}^{2+}$ ions [38].

The shorter Ti-O and larger Ti-Zn(Ti) distances at the first and second shells imply an amorphouslike local structure [39], i.e., a highly ordered local structure. The presence of some $\mathrm{TiO}_{4}$ clusters observed in $\mathrm{Cu}$ doped $\mathrm{ZnO}$ cannot be ruled out [40]. In fact, the boundary for substitutional doping in $\mathrm{ZnO}$ and clustering becomes ambiguous if the intermediate range ordering is missing. The substitution of $\mathrm{Ti}$ in the $\mathrm{Zn}$ sites has induced a large distortion in the $\mathrm{ZnO}$ lattice.

Figure 5. Theoretical fit to the first peak in the real space for (a) $\mathrm{Zn}_{0.972} \mathrm{Ti}_{0.028}$ and (b) $\mathrm{Zn}_{0.933} \mathrm{Ti}_{0.067} \mathrm{O}$ films.



Table 2. Structural parameters of references from the literatures and results of the data fit of the Ti-O or $\mathrm{Zn}-\mathrm{O}(\mathrm{ZnO})$ shell in $\mathrm{Zn}_{0.972} \mathrm{Ti}_{0.028} \mathrm{O}$ and $\mathrm{Zn}_{0.933} \mathrm{Ti}_{0.067} \mathrm{O}$ films. $\mathrm{CN}=$ coordination number; $R(\AA)=$ bond length; and $\sigma^{2}\left(\AA^{2}\right)=$ Debye-Waller factor. The uncertainties are $10 \%, 0.02 \AA$, and $10 \%$, respectively.

\begin{tabular}{lccc}
\hline \multicolumn{1}{c}{ Sample } & $\mathbf{C N}$ & $\mathbf{R}$ & $\boldsymbol{\sigma}^{\mathbf{2}}$ \\
\hline $\mathrm{ZnO}$ & 4 & 1.97 & -- \\
$\mathrm{Ti}_{2} \mathrm{O}_{3}$ & 6 & 2.05 & -- \\
Anatase $\mathrm{TiO}_{2}$ & 6 & 1.95 & -- \\
${\mathrm{Rutile} \mathrm{TiO}_{2}}_{\mathrm{Zn}_{0.972} \mathrm{Ti}_{0.028} \mathrm{O}}$ & 6 & 1.96 & -- \\
$\mathrm{Zn}_{0.933} \mathrm{Ti}_{0.067} \mathrm{O}$ & 4.3 & 1.89 & 0.0044 \\
$\mathrm{Zn}_{2} \mathrm{Ti}_{3} \mathrm{O}_{8}$ & 5.4 & 1.87 & 0.0087 \\
$\mathrm{ZnTiO}_{3}$ & 6 & 2.10 & \\
\hline
\end{tabular}


The M-H magnetization ( $\left.\mu_{B} / \mathrm{Ti}\right)$ curves measured at room temperature are shown in Figure 6 for the doped samples. All films are ferromagnetic as observed from the $\mathrm{M}-\mathrm{H}$ hysteresis loops. The saturation magnetization $\mathrm{Ms}\left(\mu_{B} / \mathrm{Ti}\right)$ values were derived and listed in Table 1 . The largest magnetic moment is $0.827 \pm 0.013 \mu_{B} / \mathrm{Ti}$ for $\mathrm{Zn}_{0.994} \mathrm{Ti}_{0.006} \mathrm{O}$, several times larger than that $\left(0.15 \mu_{B} / \mathrm{Ti}\right)$ reported by Antony et al. [13] and Venkatesan et al. [8] at room temperature for 5\% Ti-doped $\mathrm{ZnO}$ sample. This is astonishing. One can note that the two $0.6 \%$ and $0.4 \%$ samples with similar doping concentrations show a large difference in the magnetic moment. The XANES spectra have shown a majority of tetrahedral Ti-O coodination and a portion of $\mathrm{Ti}$ species with higher Ti-O coordinations in the $0.6 \%$ doped sample. However, the unusually high ferromagnetism might not be attributed to the precipitates, since none of them are ferromagnetic.

Figure 6. M-H loops measured at room temperature for Ti-doped $\mathrm{ZnO}$ films.



The Ti-doped ZnO thin film samples in the present study are insulators; hence, the hole-mediated mechanism is unlikely to interpret the observed ferromagnetism. Recent studies have shown that intrinsic defects in $\mathrm{ZnO}$ play an important role in the room-temperature ferromagnetism in transition metal doped $\mathrm{ZnO}$, in particular, with the cationic vacancies [41-43]. Similar explanations may also be applicable for the Ti-doped $\mathrm{ZnO}$ samples. High magnetic moment for the $80 \% \mathrm{Ar}, 20 \% \mathrm{O}_{2}$ ambient sample indicates the ferromagnetism may not be oxygen vacancy relevant because in an oxygen-rich atmosphere, it is not favorable for oxygen vacancy formation. The ferromagnetic ordering may be improved by the cationic vacancy-derived impurity band. Similar to $\mathrm{Li}$ doped $\mathrm{ZnO}$, the formation energy of Zn vacancies can be lowered by the doping of Ti by forming defect complexes [43]. A higher Ti valence state than $\mathrm{Zn}^{2+}$ implies that $\mathrm{Ti}$ atoms may act as donors by supplying electrons or they may favor the creation of more $\mathrm{Zn}$ vacancies (V0 centers) [12]. It is clear that more experiments and theoretical calculations are necessary to provide direct evidence.

\section{Conclusions}

$\mathrm{Zn}_{1-x} \mathrm{Ti}_{x} \mathrm{O}(\mathrm{x}=0.004,0.006,0.028,0.067)$ films were fabricated on Si substrates by radio-frequency magnetron sputtering. Ferromagnetism was measured at room temperature. A strong ambient condition 
dependence of the structures and magnetism was observed; the film sample prepared at $80 \% \mathrm{Ar}, 20 \% \mathrm{O}_{2}$ ambient shows the largest magnetic moment $\left(0.827 \pm 0.013 \mu_{B} / \mathrm{Ti}\right)$. The local environment of Ti was investigated by measuring XAFS at Ti K-edge. The results indicate that the majority of Ti ions take the tetrahedral crystallographic sites and are incorporated into the substitutional sites in the ZnO lattice. At higher concentration doping (6.7\%), some precipitates of Ti-O speciation may exist. The FT spectra and fit results indicate a strong Ti-O interaction and the Ti doping induces a large distortion because of the lattice mismatch. The preparation at different ambient conditions may change charge equilibrium in the Ti-doped $\mathrm{ZnO}$ and hence affect the $\mathrm{Zn}$ vacancy concentration.

\section{Acknowledgements}

This work was supported by A*STAR/MOE RP3979908M grant, A*STAR 0121050038 grant, NUS Core Support C-380-003-003-001 grant, Research Fellowship from A*STAR grant R-144-000106-305, NRF-CRP grant “Tailoring Oxide Electronics by Atomic Control” (NRF2008NRF-CRP002024), NUS YIA, NUS grant, cross faculty grant and FRC grant.

\section{References}

1. Murakami, M.; Matsmoto, Y.; Hasegawa, T. Cobalt valence states and origins of ferromagnetism in Co doped $\mathrm{TiO}_{2}$ rutile thin films. J. Appl. Phys. 2004, 95, 5330-5333.

2. Jalbout, A.F.; Chen, H.; Whittenburg, S.L. Monte Carlo simulation on the indirect exchange interactions of Co-doped ZnO film. Appl. Phys. Lett. 2002, 81, 2217-2219.

3. Sato, K.; Katayama-Yoshida, H. Material design for transparent ferromagnets with ZnO-based magnetic semiconductors. Jpn. J. Appl. Phys. Part 2 2001, 40, L334-L336.

4. Coey, J.M.D.; Chambers, S.A. Oxide dilute magnetic semiconductors-fact or fiction. Mater. Res. Bull. 2008, 33, 1503-1058.

5. Coey, J.M.D. Dilute magnetic oxides. Curr. Opin. Solid State Mater. Sci. 2007, 10, 83-92.

6. Chambers, S.A. Ferromagnetism in doped thin-film oxide and nitride semiconductors and dielectrics. Surf. Sci. Rep. 2006, 61, 345-381.

7. Coey, J.M.D.; Venkatesan, M.; Fitzgerald, C.B. Donor impurity band exchange in dilute ferromagnetic oxides. Nat. Mater. 2005, 4, 173-179.

8. Venkatesan, M.; Fitzgerald, C.B.; Lunney, J.G.; Coey, J.M.D. Anisotropic ferromagnetism in substituted zinc oxide. Phys. Rev. Lett. 2004, 93, 177206.

9. Song, C.; Geng, K.W.; Zeng, F.; Wang, X.B.; Shen, Y.X.; Pan, F.; Xie, Y.N.; Liu, T.; Zhou H.T.; Fan, Z. Local Co structure and ferromagnetism in ion-implanted Co-doped $\mathrm{LiNbO}_{3}$. Phys. Rev. B 2006, 73, 024405.

10. Elfimov, I.S.; Yunoki, S.; Sawatzky, G.A. Possible path to a new class of ferromagnetic and halfmetallic ferromagnetic materials. Phys. Rev. Lett. 2002, 89, 216403.

11. Osorio-Guillén, J.; Lany, S.; Zunger, A. Atomic control of conductivity versus ferromagnetism in wide-gap oxides via selective doping: $\mathrm{V}, \mathrm{Nb}$, Ta in anatase $\mathrm{TiO}_{2}$. Phys. Rev. Lett. 2008, 100, 036601.

12. Xiong, Z.; Jiang, F. First-principles study of electronic structure and ferromagnetism in Ti-doped ZnO. J. Phys. Chem. Solids 2007, 68, 1500-1503. 
13. Antony, J.; Pendyala, S.; McCready, D.E.; Engelhard, M.H. Ferromagnetism in Ti-doped ZnO nanoclusters above room temperature. IEEE Trans. Magn. 2006, 42, 2697-2699.

14. Osuch, K.; Lombardi, E.B.; Gebicki, W. First principles study of ferromagnetism in $\mathrm{Ti}_{0.0625} Z \mathrm{n}_{0.9375} \mathrm{O}$. Phys. Rev. B 2006, 73, 075202.

15. Sato, K.; Katayama-Yoshida, H. Ferromagnetism in a transition metal atom doped ZnO. Physica E 2001, 10, 251-255.

16. Hartnagel, H.L.; Dawar, A.L.; Jain, A.K. Semiconducting Transparent Thin Films; Institute of Physics Publishing: Bristol, UK, 1995.

17. Jeong, S.; Kim, I.; Kim, J.; Lee, B. Quality improvement of ZnO layer on LT-grown ZnO layer/Si(111) through a two-step growth using an RF magnetron sputtering. J. Cryst. Growth 2004, 264, 327-333.

18. Yan, J.F.; Lua, Y.M.; Liu, Y.C.; Liang, H.W.; Li, B.H.; Shen, D.Z.; Zhang, J.Y.; Fan, X.W. Improvement of the crystalline quality of the $\mathrm{ZnO}$ epitaxial layer on a low-temperature grown ZnO buffer layer. J. Cryst. Growth 2004, 266, 505-510.

19. Park, T.E.; Kong, B.H.; Cho, H.K.; Park, D.J.; Lee, J.Y. Influence of gas atmosphere during growth interruption in the deposition of $\mathrm{ZnO}$ films by magnetron sputtering. Physica B 2006, 376, 735-740.

20. Yeo, A.; Yang, P.; Fane, A.G.; White, T.; Moser, H.O. Non-invasive observation of external and internal deposition during membrane filtration by X-ray microimaging (XMI). J. Membrane Sci. 2005, 250, 189-193.

21. Liu, T.; Gao, X.Y.; Wee, A.T.S.; Ping, Y.; Moser, H.O. Study of thin films and aqueous chlorine solution using X-ray absorption fine structure spectroscopy. Adv. Synchrotron Radiat. 2008, 1, 79-87.

22. Uruga, T.; Tanida, H.; Yoneda, Y.; Takeshita, K.; Emura, S.; Takahashi, M. The XAFS beamline BL01B1 at SPring-8. J. Synchrotron Radiat. 1999, 6, 143-145.

23. Nishihata, Y.; Emura, S.; Maeda, H.; Kubozono, Y.; Harada, M.; Uruga, T.; Tanida, H.; Yoneda, Y.; Mizuki, J.; Emoto, T. XAFS spectra in the high-energy region measured at SPring-8. J. Synchrotron Radiat. 1999, 6, 149-151.

24. Tanaka, I.; Mizoguchi, T.; Matsui, M.; Yoshioka, S.; Adachi, H.; Yamamoto, T. Identification of ultradilute dopants in ceramics. Nat. Mater. 2003, 2, 541-545.

25. Herng, T.S.; Lau, S.P.; Yu, S.F.; Yang, H.Y.; Ji, X.H.; Chen, J.S.; Yasui, N.; Inaba, H. Origin of room temperature ferromagnetism in ZnO: Cu films. J. Appl. Phys. 2006, 99, 086101:1-086101:3.

26. Bashi, Z.B.; Oral, A.Y. Effects of $\mathrm{Mn}$ and $\mathrm{Cu}$ Edoping on the microstructures and optical properties of sol-gel derived ZnO thin films. Opt. Mater. 2007, 29, 672-678.

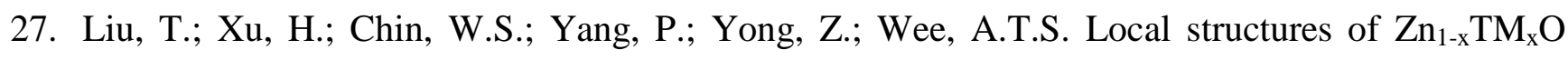
( $\mathrm{TM}=\mathrm{Co}, \mathrm{Mn}$, and $\mathrm{Cu}$ ) nanoparticles studied by $\mathrm{X}$-ray absorption fine structure spectroscopy and multiple scattering calculations. J. Phys. Chem. C 2008, 112, 13410-13418.

28. Han, A.R.; Hwang, S.; Zhao, Y.; Kwon, Y. X-ray absorption spectroscopic and magnetic characterization of cobalt-doped zinc oxide nanocrystals prepared by the molten-salt method. $J$. Magn. Magn. Mater. 2008, 320, 1591-1596. 
29. Burdett, J.K.; Hughbanks, T.; Miller, G.J.; Richardson, J.W., Jr.; Smith, J.V. Structural electronic relationships in inorganic solids-powder neutron-diffraction studies of the Rutile and Anatase polymorphs of Titanium-dioxide at 15 and 295-K. J. Am. Chem. Soc. 1987, 109, 3639-3646.

30. Rice, C.E.; Robinson, W.R. Structural-changes associated with semiconductor-to-metal transition in $\mathrm{Ti}_{2} \mathrm{O}_{3}$. Mat. Res. Bull. 1976, 11, 1355-1359.

31. Joly, Y.; Cabaret, D.; Renevier, H.; Natoli, C.R. Electron population analysis by full-potential Xray absorption simulations. Phys. Rev. Lett. 1999, 82, 2398-2401.

32. Waychunas, G.A. Synchrotron radiation XANES spectroscopy of $\mathrm{Ti}$ in minerals-effects of $\mathrm{Ti}$ bonding distances, Ti valence, and site geometry on absorption-edge structure. Amer. Mineral. 1987, 72, 89-101.

33. Farges, F.; Brown, G.E., Jr.; Rehr, J.J. Ti K-edge XANES studies of Ti coordination and disorder in oxide compounds: Comparison between theory and experiment. Phys. Rev. B 1997, 56, 1809-1819.

34. Shi, T.; Zhu, S.; Sun, Z.; Wei, S.; Liu, W. Structures and magnetic properties of wurtzite $\mathrm{Zn}_{1-\mathrm{x}} \mathrm{Co}_{\mathrm{x}} \mathrm{O}$ dilute magnetic semiconductor nanocomposites. Appl. Phys. Lett. 2007, 90, 102108.

35. Shannon, R.D.; Prewitt, C.T. Effective ionic radii in oxides and fluorides. Acta Cryst. 1969, 825, 925-946.

36. Liu, T.; Xu, H.; Chin, W.S.; Yong, Z.; Wee, A.T.S. Local structural evolution of Co-doped ZnO nanoparticles upon calcination studied by in situ quick-scan XAFS. J. Phys. Chem. C 2008, 112, 3489-3495.

37. Steinike, U.; Wallis, B. Formation and structure of Ti-Zn-oxides. Cryst. Res. Tech. 1997, 32, 187-193.

38. Bartram, S.F.; Slepetys, A. Compound formation and crystal structure in the system $\mathrm{ZnO}-\mathrm{TiO}_{2}$. $J$. Am. Ceram. Soc. 1961, 44, 493-499.

39. Liu, X.J.; Chen, G.L.; Hui, X.; Liu, T.; Lu, Z.P. Ordered clusters and free volume in a Zr-Ni metallic glass. Appl. Phys. Lett. 2008, 93, 011911.

40. Ma, Q.; Buchholz, D.B.; Chang, R.P.H. Local structures of Copper-doped ZnO films. Phys. Rev. B 2008, 78, 214429.

41. Sluiter, M.H.F.; Kawazoe, Y.; Sharma, P.; Inoue, A.; Raju, A.R.; Rout, C.; Waghmare, U.V. First principles based design and experimental evidence for a $\mathrm{ZnO}$-based ferromagnet at room temperature. Phys. Rev. Lett. 2005, 94, 187204.

42. Yan, W.; Sun, Z.; Liu, Q.; Li, Z.; Pan, Z.; Wang, J.; Wei, S.; Wang, D.; Zhou, Y.; Zhang, X.; Zn vacancy induced room-temperature ferromagnetism in Mn-doped ZnO. Appl. Phys. Lett. 2007, 91, 062113.

43. Yi, J.B.; Lim, C.C.; Xing, G.Z.; Fan, H.M.; Van, L.H.; Huang, S.L.; Yang, K.S.; Huang, X.L.; Qin, X.B.; Wang, B.Y.; et al. Ferromagnetism in dilute magnetic semiconductors through defect engineering: Li-doped ZnO. Phys. Rev. Lett. 2010, 104, 13720.

(C) 2010 by the authors; licensee MDPI, Basel, Switzerland. This article is an Open Access article distributed under the terms and conditions of the Creative Commons Attribution license (http://creativecommons.org/licenses/by/3.0/). 